\title{
An Electronic Prosthesis Mimicking the Dynamic Vestibular Function
}

\author{
Jiayin Liu \\ Mechanical and Aerospace \\ Engineering Dept. \\ University of California at Irvine \\ Irvine, CA, USA \\ jiayinl@uci.edu
}

\author{
Andrei M. Shkel \\ Mechanical and Aerospace \\ Engineering Dept. \\ Biomedical Engineering Dept. \\ University of California at Irvine \\ Irvine, CA, USA \\ ashkel@uci.edu
}

\begin{abstract}
An analog circuit has been designed to mimic the dynamic function of a unilateral semicircular canal of the vestibular system. The circuitry is an integral part of a novel MEMS-based electrostimulatory prosthesis currently investigated by this research group [1]. The device is intended for restoration of permanently damaged balance function. The present experimental results indicate that under the same inertial loading conditions it is feasible to design a prosthesis that matches the signal recorded from the vestibular nerve in squirrel monkey experiments reported in the literature [4].
\end{abstract}

\section{Keywords}

Neural vestibular prosthesis, artificial implants, inertial MEMS applications

\section{INTRODUCTION}

The vestibular system of the inner ear is the major anatomical system to sense motion. The primary function of the vestibular system is to provide information to the brain about the body's motion and orientation. It measures six quantities concerning the spatial orientation including 3-axes angular acceleration and 3-axes linear acceleration. Diminished balance ability, often seen in older adults, poses a serious health risk due to the increased likelihood of the falling.

Several approaches can be considered for improvement of balance sensation and postural control. Appropriate drugs could relief some of the symptoms. Non-invasive exercise has relatively low risk and may also lead to improvement of the vestibular function. If the natural tactile sensitivity and balance control cannot be improved using non-invasive methods, prosthesis might be an alternative way to restore balance function. In balance related prosthetics, sensors such as gyroscopes and accelerometers can sense the motion and provide patients with information about body's orientation. Such a device could be used as a temporary aid during recovery from ablative inner-ear surgery and as a permanent prosthesis for those elderly prone to falls [5].

Vestibular prosthesis could be either implantable or nonimplantable. Implantable prosthesis interfaces with the nervous system directly and stimulate the neural pathways related to spatial orientation. Non-implantable prosthesis provides the information via sensory substitution through other sensory systems, such as tactile, visual, auditory system [?].

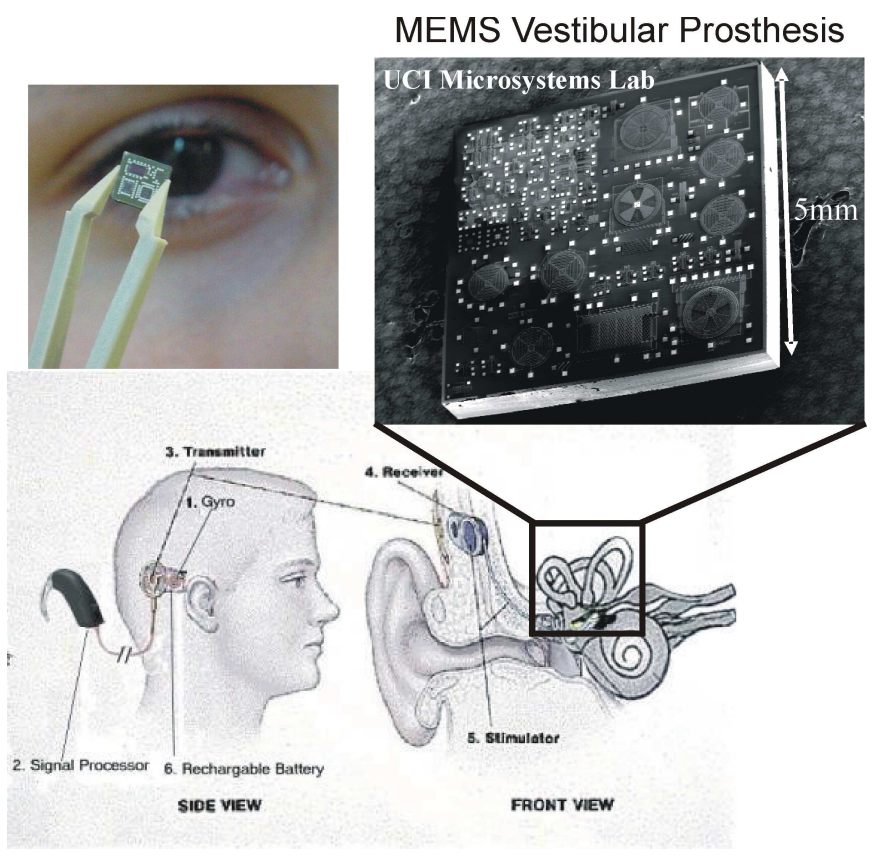

Figure 1. Conceptual design of the MEMS based vestibular prosthesis

Our research falls into the category of implantable prosthesis. It offers potential opportunity to restore seriously diminished vestibular systems. The long term goal of this project is to explore opportunities for development of a totally implantable MEMS-based electronic device which functions identically to the vestibular system, Figure 1. Using unique feature of the MEMS technology and Integrated Circuit (IC) technology, micromachining can shrink the sensors size by orders of magnitude, reduce the fabrication cost significantly, and allow the electronics to be integrated on the same silicon chip [7]. Figure 1 shows a 5 by 5 millimeter silicon chip developed by UCI's Micro-system lab. The chip in- 
cludes over 10 gyroscopes and several linear and angular accelerometers. By merging two advanced technologies of micro-machined gyroscope [7] and cochlear implants [6], it is possible to build a micro-vestibular neural prosthetics which restores balance function, consumes little power, and can be made in batches. Potentially, the ensemble of six inertial MEMS sensors required to measure the six-degrees of freedom of head motion can be built on a single silicon chip and packaged in a volume smaller than 1 cubic centimeter. Review on the technology of inertial sensors on-a-chip can be found in [8].

\section{SYSTEM DESIGN}

Figure 2 presents the functional blocks of the circuit components of a vestibular implant. The electronics include a sensing unit, a pulse generating unit, and a current stimulator [4]. The sensing unit includes a gyroscope, a low-pass filter and a differentiator; it detects the motion of the head and sends out the analog voltage signal proportional to angular acceleration about the sensing axis. The pulse generator consists of a transfer function unit and a voltage-to-frequency converter; it generates monophasic voltage pulses based on a selected mathematical model. The current source includes monophasic-to-biphasic converter (Smith triggers and analog switches) and a current mirror; they convert the monophasic voltage pulses to biphasic, chargebalanced, cathodic-first current pulses which can be used to stimulate vestibular neurons.

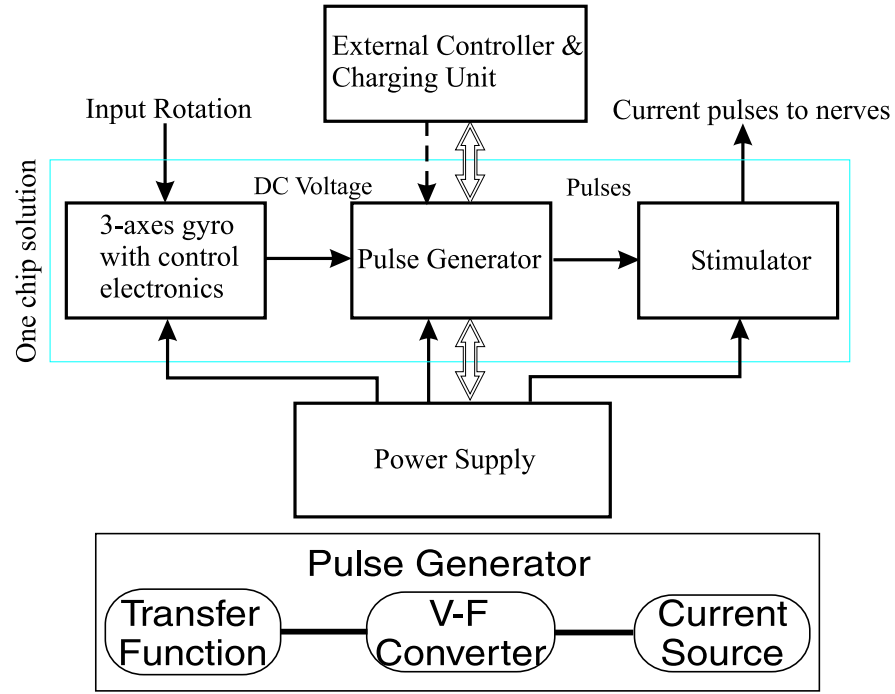

Figure 2. Electronic prosthesis mimicking the natural vestibular system

\section{Sensing Unit}

Humans detect orientation using the semicircular canals and linear motion using the Otolith organs (utricle and saccule). These organs have small sensory hair cells that send pulses to the brain, where information about head

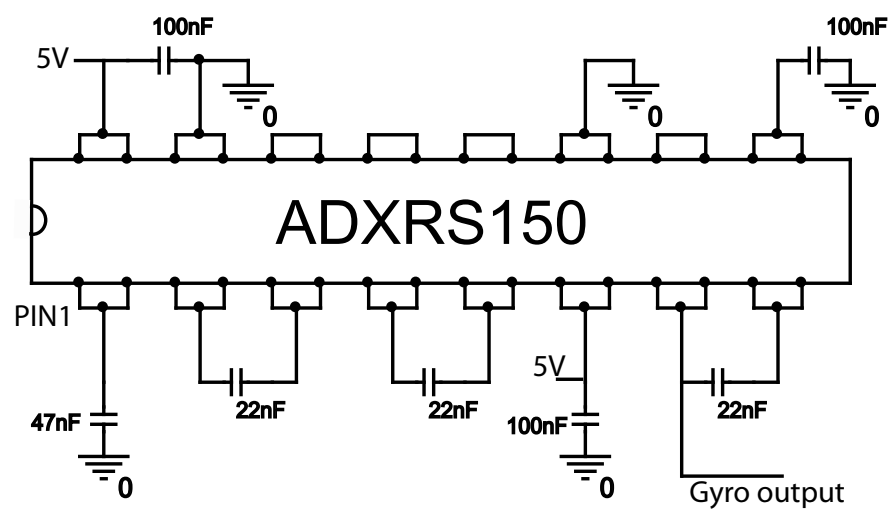

(a)

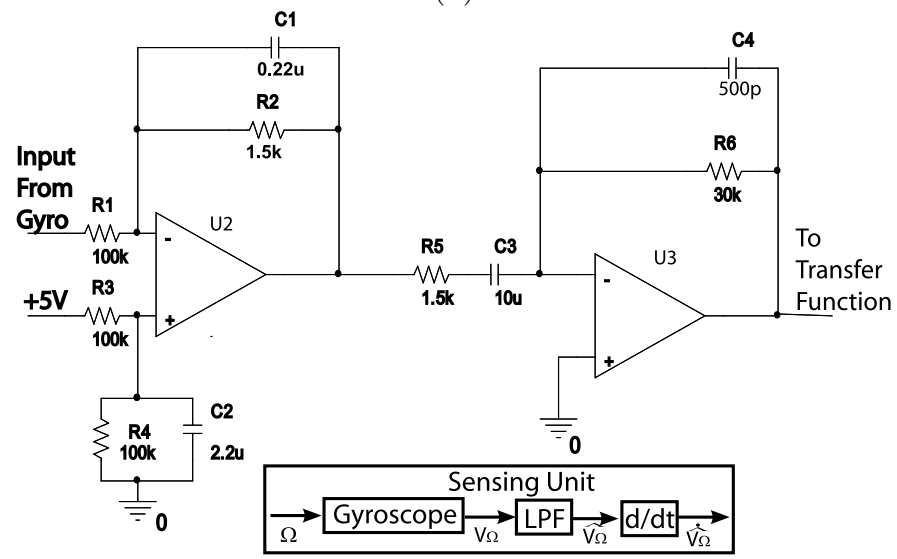

(b)

Figure 3. Sensing Unit includes: (a) gyroscope and (b) low-pass filter and differentiator

movement is combined with information from the eyes, muscles, and joints. Similarly, MEMS gyroscopes and accelerometers are used to detect rotation and linear motion.

Our prototype of a unilateral semicircular prosthesis, Figure 3, includes a gyroscope, a low-pass filter and a differentiator (for the demonstration purposes, we were using a commercial prototype of a single axis gyroscope (Model ADXRS150)). This device is built using integrated polysilicon surface micromachining technology, with electronics integrated on the same chip. In order to mimic the response of the natural vestibular endorgan, responding to angular accelerations, the output voltage of the gyroscope, which is proportional to the angular velocity, need to be differentiated to produce a signal similar to the natural organ - angular acceleration. In our implementation, a low-pass filter is utilized before the differentiator to minimize the effect of highfrequency noise, [1].

\section{Pulse Generator}

Voltage signal from the sensing unit is transmitted to a pulse generator, and consequently to a current source. The pulse generator consists of a transfer function unit 
based on the mathematical model of the vestibular system and a Voltage-to-Frequency converter, which converts voltage signals to frequency coded pulses.

In the linear approximation, the transfer function emulating dynamics of the natural vestibular organs defines the relationship between the input angular acceleration and the change in the output of the pulse rate

$$
H(s)=\frac{\tau_{A} s}{1+\tau_{A} s} \cdot \frac{1+\tau_{L} s}{\left(1+\tau_{1} s\right)\left(1+\tau_{2} s\right)}
$$

where $\tau_{1}$ and $\tau_{2}$ are two time constants of the pendulum model, $\tau_{A}$ is related to the level of neuron adaptability, and $\tau_{L}$ is the dynamical-electrical time constant. Experimentally obtained results from the squirrel monkey model [2], estimated that $\tau_{1}=5.7 \mathrm{sec}, \tau_{2}=0.003 \mathrm{sec}$, $\tau_{A}=80 \mathrm{sec}$, and $\tau_{L}=0.049 \mathrm{sec}$. These values are used in the circuit design of the pulse generating unit.

\section{Current Source}

The current pulses in the neural network are delivered by ion flow, and the overall charge sent to brain should be zero [6], i.e. the integration of the stimulation current over time should be zero. Since tissue impedance is always changing, a voltage source may not maintain constant current for charge delivery. Thus, a current source, rather than a voltage source, is required to stimulate neurons.

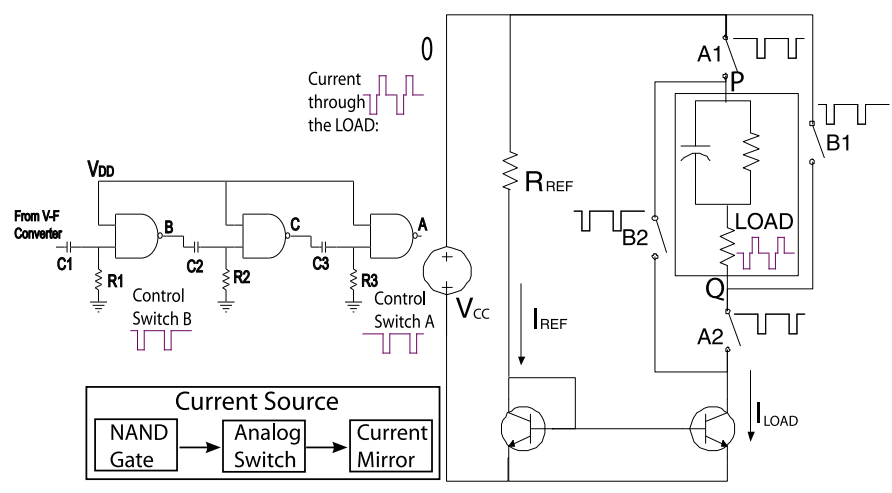

Figure 4. Current source converting monophasic voltage pulses to biphasic current pulses

Figure 4 shows our conceptual implementation of the current source, which includes a current mirror, analog switches and Smith triggers. The analog switches and Smith triggers convert monophasic signal to biphasic signal, and the current mirror provides current output that is not affected by the load impedance. The electrical properties of a biological tissue can be modelled by an equivalent circuit which includes a resistor and a capacitor in parallel, plus a resistor in series. The values of the resistors and capacitors may fluctuate. By using the current mirror, the voltage across the LOAD may change due to impedance changes of the tissue.
However, the current through the LOAD will not be affected.

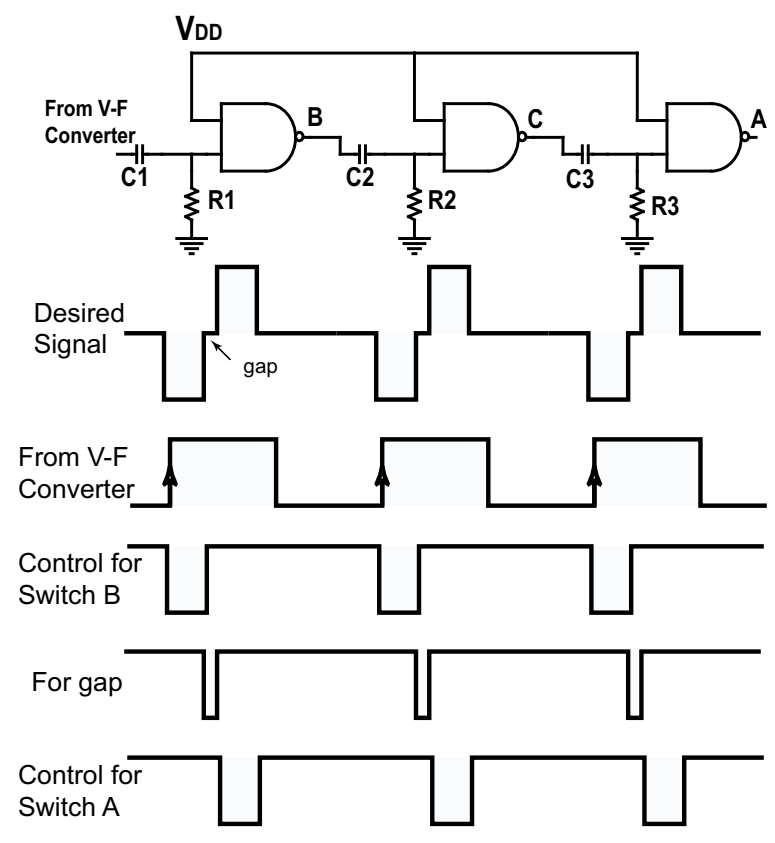

Figure 5. Control signal of the switches using Smith triggers

The Pulse Duration (PD) is defined by the time constant of each smith trigger, as long as the output voltage increases before the input voltage drops, i.e., the pulse duration is not influenced by the input frequency. For each NAND Gates, Figure 5, when one of the input is kept high, another input $\left(V_{1}\right)$ governs the output. As soon as the voltage at $V_{1}$ falls below $V_{T-}$ (1.8Volt), $V_{O U T}$ changes from low to high. So the pulse duration (PD) in $V_{O U T}$ can be calculated by

$$
e^{\frac{P D}{\tau}}=\frac{V_{D D}}{V_{1}}
$$

The maximum neuron firing rate is around $250 \mathrm{~Hz}[3]$, so the minimum duration for each firing cycle is around $4 \mathrm{~ms}$. Since the pulse duration in $V_{O U T}$ should be less than half of the firing cycle, the $\mathrm{PD}+$ and $\mathrm{PD}$ - are designed to be $1 \mathrm{~ms}$. Substituting 5 Volt in $V_{D D}$ and 1.8 Volt in $V_{T-}$, time constant $\tau$ can be calculated from Equation (2)

$$
\tau=\frac{P D}{\ln \left(\frac{V_{D D}}{V_{1}}\right)}=\frac{P D}{\ln \left(\frac{5}{1.8}\right)}=1.0217
$$

Three Smith triggers are used in our implementation to achieve control signal for the switches. In Figure 5, the rising edge from the output of the voltage-to-frequency converter triggers a negative pulse at point $\mathrm{A}$, the rising edge from the voltage at point $\mathrm{A}$ triggers a negative pulse at point $\mathrm{B}$, and the rising edge from point $\mathrm{B}$ triggers a negative pulse at point $\mathrm{C}$. The pulse duration is defined by the time constant of each Smith trigger, as 
long as the output voltage increases before the input voltage drops. Control signals at point $A$ and point $B$ determine the state of analog switches, $\mathrm{A}$ and $\mathrm{B}$, respectively. Using the scheme, the biphasic current pulses are generated and are kept constant during the stimulation. The proposed scheme allows to adjust pulse durations (e.g., if desired by an experiment on different subjects). The pulse frequency can be adjusted by changing resistances $R_{1}$ or $R_{3}$, Figure 5 . The pulse magnitude can be adjusted by modifying $R_{R E F}$, Figure 4 .

\section{EXPERIMENTAL RESULTS}

Based on the discussed design, a circuitry prototype is implemented on a Printed Circuit Board (PCB), Figure 6. It consists of a sensing unit which includes a z-axis gyroscope, a low-pass filter and a differentiator; a pulse generating unit which includes a transfer function unit and a voltage-to-frequency converter, and a current source which includes Smith triggers, analog switches and a current mirror. Two nine volt batteries are used as a power supply for the circuitry and the sensor. Nine potentiometers are utilized to adjust for the resistance parameters, including four time constants in the transfer function $\left(\tau_{A}, \tau_{1}, \tau_{2}\right.$, and $\left.\tau_{L}\right)$, rest firing rate, gain of the transfer function, positive and negative pulse duration, and magnitude of the current pulse.

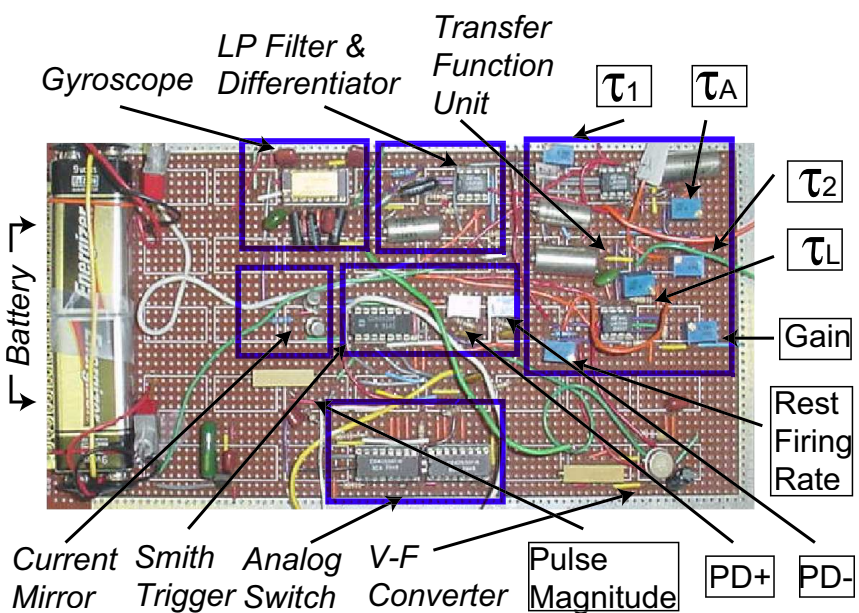

Mirror Trigger Switch Converter Magnitude

\begin{tabular}{|c|c|c|}
\hline \begin{tabular}{|l|} 
Gyroscope \\
Low-pass filter \\
Differentiator
\end{tabular} & $\begin{array}{l}\text { Transfer function unit } \\
\text { Voltage-to-frequency }\end{array}$ & $\begin{array}{l}\text { Monophasic-to-biphasid } \\
\text { converter } \\
\text { Current mirror }\end{array}$ \\
\hline Sensing Unit & Pulse Generator & Current Source \\
\hline
\end{tabular}

Figure 6. Printed Circuit Board (PCB) prototype of the electrostimulatory unilateral prosthesis

\section{Time Domain Response}

A fragment of the circuit response to sinusoidal voltage input is illustrated in Figure 7 at three different time scales $(500 \mathrm{~ms}, 50 \mathrm{~ms}, 2 \mathrm{~ms}$ in (a), (b), (c), correspondingly). The voltage response is recorded using Virtual

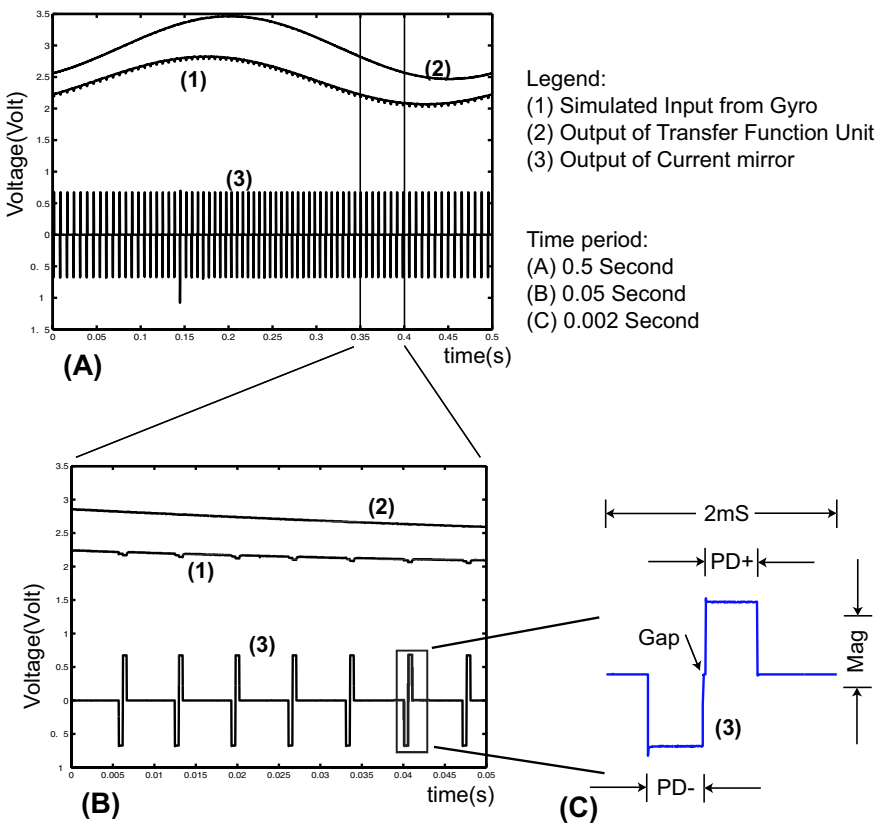

Figure 7. Circuitry response to harmonic inertial stimuli

Bench 2.1.1 (a Labview program by National Instruments) through a data acquisition board (National Instruments BNC-2110). Then the data was saved as the text file, which was subsequently imported and analyzed in Matlab.

Legend (1) in Figure 7 is generated from a signal generator at $2 \mathrm{~Hz}$ frequency, $0.5 \mathrm{~V}$ peak amplitude, and $2.43 \mathrm{Volt}$ offset, and it acts as the output from the gyroscope. The gyro calibration shows that the corresponding motion is harmonic rotation with zero offset, $2 \mathrm{~Hz}$ frequency and 40degree/second peak amplitude. Legend (2) shows the voltage signal after the transfer function unit, and it is proportional to the pulse rate of legend (3), Figure 7 (a). For the convenience of recording, voltage across the load (Legend 3) is measured. The current through the load is obtained by dividing the voltage by the load impedance, which in turns consists of biphasic, charge-balanced, and cathode-first pulses. Changing the load impedance, the amplitude of voltage pulses changes, and the amplitude of the current pulses is kept constant. Figure 7 (b) and (c) scale the time duration down to $50 \mathrm{~ms}$ and $2 \mathrm{~ms}$, correspondingly. Figure 7 (c) illustrates the voltage pulses across the load. Three parameters of the pulse shape can be adjusted for the best performance of the stimulator, i.e., positive and negative pulse duration ( $\mathrm{PD}+, \mathrm{PD}-)$ and pulse magnitude (Mag).

\section{Gain and Phase Response in Frequency Domain}

To obtain more precise gain and phase response, the circuitry is swept by a signal analyzer between frequencies $0.1 \mathrm{~Hz}$ and $10 \mathrm{~Hz}$, Figure 8. Data points marked 

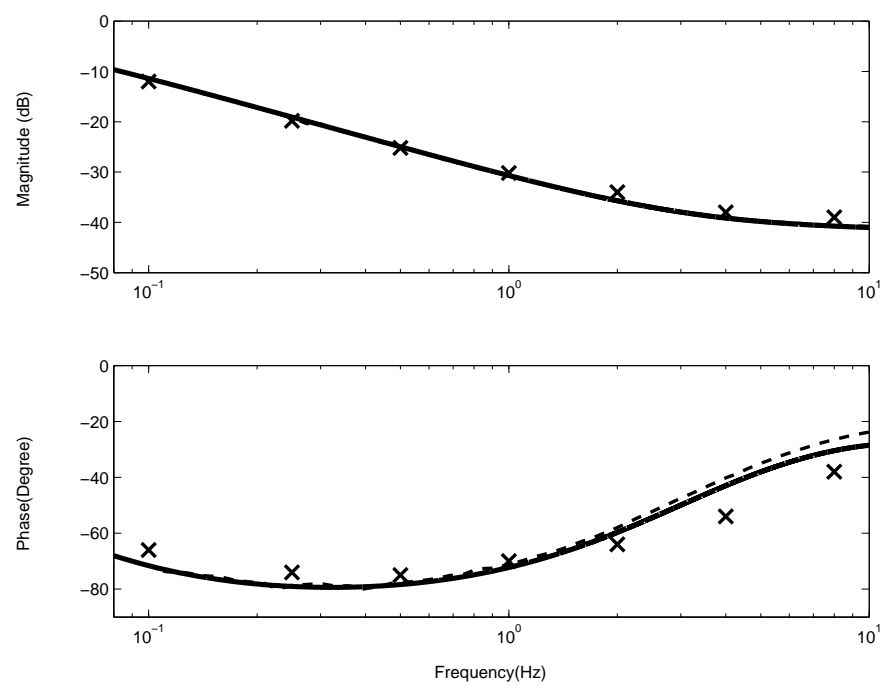

Figure 8. Comparison of response of the circuitry and of the natural vestibular system in the squirrel monkey experiments reported in the literature [2]

with $(\mathrm{X})$ represent experimentally measured responses in firing of nerves in semicircular canals of the squirrel monkey animal model [2]. The continuous line illustrates the mathematical model and the dashed line is the data from the response of the prosthesis. The circuit response matches well the mathematical model, Figure 8.

Figure 8 demonstrates a very close match between the response of the circuitry and the natural vestibular organ. The slight mismatch between the response from the circuitry and the natural nerve can be possibly minimized by adjusting the time constant in the transfer function.

\section{Parameter Sensitivity}

Figure 9 illustrates a bode plot with asymptotes of the mathematical model $\mathrm{H}(\mathrm{s})$. The continuous line is the actual response of the mathematical model in the frequency domain. The four time constants characterize the break points of the gain and the phase, defined by dashed asymptotes, Figure 9 . The value of the four time constants are shown in the transfer function $\mathrm{H}(\mathrm{s})$

$$
\begin{aligned}
H(s)= & \frac{\tau_{A} s}{1+\tau_{A} s} \frac{1+\tau_{L} s}{\left(1+\tau_{1} s\right)\left(1+\tau_{2} s\right)}= \\
& \frac{80 s}{1+80 s} \frac{1+0.049 s}{(1+5.7 s)(1+0.003 s)}
\end{aligned}
$$

In our implementation, $1 / \tau_{A}, 1 / \tau_{1}, 1 / \tau_{2}$, and $1 / \tau_{L}$ are equal to $0.0125 \mathrm{rad} / \mathrm{s}, 0.1754 \mathrm{rad} / \mathrm{s}, 333.3 \mathrm{rad} / \mathrm{s}$, and $20.42 \mathrm{rad} / \mathrm{s}$, correspondingly.

The time constants in the denominator of the transfer function cause the magnitude slope of the asymptotes to decrease by $20 \mathrm{~dB} /$ decade and for the phase to decrease by 90 degree from the breaking point.

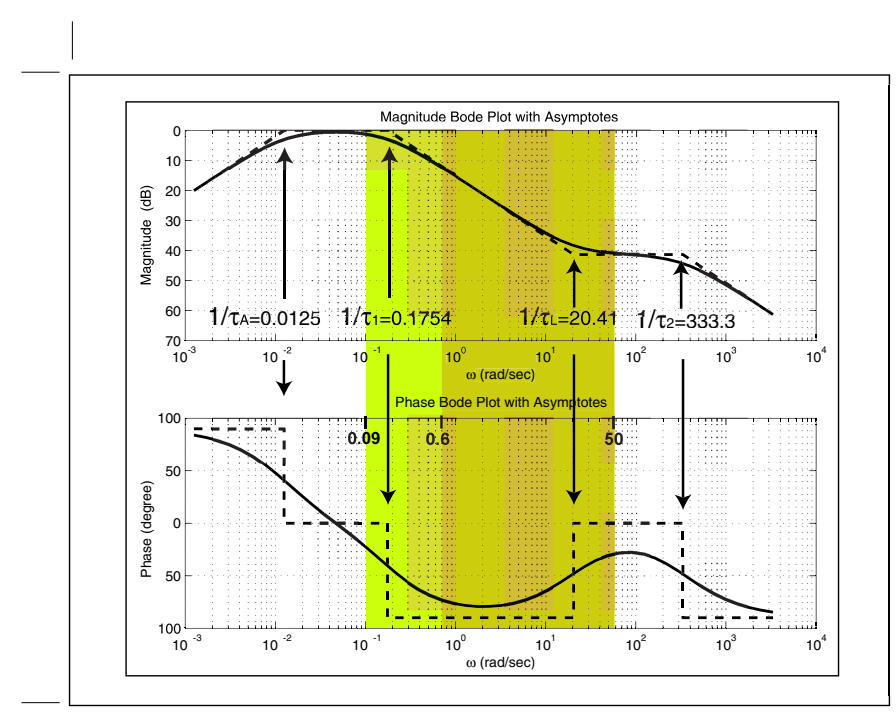

Figure 9. Bode plot with asymptotes of the mathematical model

Figure 10 illustrates how the four time constants affect the gain and phase response in the frequency range between $0.0125 \mathrm{~Hz}$ and $8 \mathrm{~Hz}(0.09$ and $50 \mathrm{rad} /$ second). Based on the experimental result reported in the reference [2], $75 \%, 90 \%, 100 \%, 110 \%$, and $125 \%$ of the original values of the four time constants are analyzed. The first break point from the left is $1 / \tau_{A}=1 / 80=$ $0.0125 \mathrm{rad} / \mathrm{sec}=1.99 \mathrm{mHz}$, which is much smaller than the frequencies tested in animal experiments. It does not affect the circuit response in tested frequency range, which can be observed in the first row in Figure 10 . This time constant can be used to adjust the response corresponding to step input instead of harmonic input. The second break point from the left is $1 / \tau_{1}=1 / 5.7=$ $0.1754 \mathrm{rad} / \mathrm{sec}=23 \mathrm{mHz}$ which lies on the left side of the tested frequency range, both gain and phase responses are largely affected by $\tau_{1}$ in tested frequency range. If $\tau_{1}$ increases by $10 \%$, the log gain decreases by around 0.04. When the input frequency is larger than $23 \mathrm{mHz}$, the phase increases by around 2.5 degree, and the input frequency falls between $2.3 \mathrm{mHz}$ and $230 \mathrm{mHz}$. The magnitude and phase response with different $\tau_{1}$ value is shown in the second row in Figure 10.

The third row in Figure 10 illustrates how the magnitude and phase response of the circuitry changes with respect to the time constant $\tau_{L}$, with the corresponding break point at $1 / \tau_{L}=1 / 0.049=20.4 \mathrm{rad} / \mathrm{sec}=$ $3.25 \mathrm{~Hz}$. If $\tau_{L}$ increases by $10 \%$, the $\log$ gain decreases around 0.04 , with the frequency larger than $3.25 \mathrm{~Hz}$. The phase response approximately increases by 2.5 degree, where the input frequency falls between $0.32 \mathrm{~Hz}$ and $32 \mathrm{~Hz}$.

The break point on the right side is $1 / \tau_{2}=1 / 0.003=$ $333 \mathrm{rad} / \mathrm{sec}=53 \mathrm{~Hz}$. This affects the magnitude and phase response in the higher frequency than the tested 

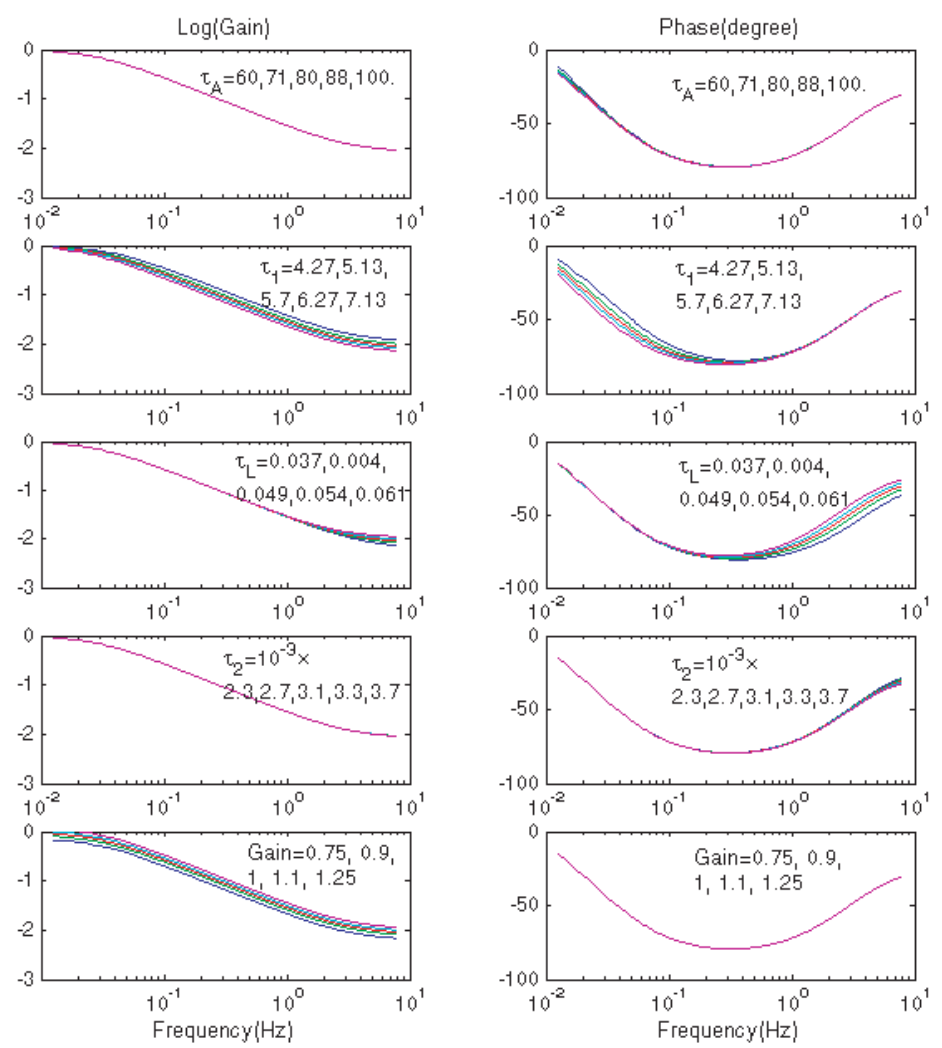

Figure 10. Bode plot shift corresponding to time constants.

frequency. The forth row in Figure 10 demonstrates that this time constant does not cause the response to change much in the tested frequency range.

In additional to adjusting the four time constants, the implementation allows to adjust gains as well. The gain does not affect phase, while if the overall gain increases by $10 \%$, the $\log$ gain increases on the same amount (around 0.04) everywhere. The related changes in the magnitude and phase response are illustrated in the last row in Figure 10.

In different animals or individuals, the response of the natural vestibular system under the same inertial condition may be different. The proposed architecture of the prosthesis allows to adjust parameters, making the response of the circuit to fit the response of the natural vestibular system.

\section{CONCLUSION}

This paper discusses implementation and experimental verification of the electronics mimicking the function of a unilateral semicircular canal. A close match between the designed electronics and response of the natural vestibular organ has been demonstrated in this paper. The paper also verifies robustness of the implemen- tation. Our next step is to demonstrate on live animal models that damaged, or temporarily disabled, vestibular organ can be effectively replaced by the electrostimulatory prosthesis with the proposed architecture. The surface mount hybrid techniques will be initially used to reduce the size of the overall system, followed by implementation of the system on a single chip. Low power consumption electronics designs are also explored for the next generation of the vestibular prosthesis. A totally implantable version of the prototype is currently explored by this group. A new system components are planned to be added to the next generation prototype, including wireless communication capabilities, wireless gain adjustment capabilities, and wireless power supply.

\section{ACKNOWLEDGEMENTS}

This project was partially supported by UC MEXUSCONACYT Grant for Collaborative Projects, Grant Number CN-02-70.

\section{REFERENCES}

[1] Andrei M. Shkel, Jiayin Liu, Chris Ikei, Fan-Gang Zeng. System Design and Experimental Evaluation of a MEMS-based Semicircular Canal Prosthesis. Conference on Neural Engineering, Vol. 1, Mar 2002, pp. 177-180.

[2] J. M. Goldberg and C. Fernandez. Physilology of Peripheral Neurons Innervating Semicircular Canals of the Squirrel Monkey. II. Response to Sinusoidal stimulation and Dynamics of Peripheral Vestibular System. J. Neurophysiol, vol.34, 1971, pp: 661-675.

[3] Wangsong. Gong and Daniel.M. Merfield. System Design and Performance of a Unilateral Horizontal Semicircular Canal Prosthesis. IEEE Transactions on Biomedical Engineering, vol.49,Feb. 2002, pp. 175-181.

[4] Jiayin Liu, Andrei M. Shkel, Kaibao Nie, Fan-Gang Zeng. IEEE Neuroengineering. Circuit with adjustable parameters mimicking function of the national vestibular end-organ., 2003.

[5] Wall, C., Weinberg, M.S.. Balance prostheses for postural control. J Neurophysiol Vol22(2), Mar. 2003, pp84-90

[6] S.U. Ay, F.-G. Zeng, B.J. Shen. Hearing with Bionic Ears. IEEE Circuits and devices Vol5, 1997, pp18-23

[7] A. M. Shkel and R. T. Howe. Micro-machined angle-measuring gyroscope. U.S. Patent 6,482,285, Mar, 2001.

[8] A. M. Shkel. Micromachined Gyroscopes: Challenges, Design Solutions, and Opportunities. 2001 SPIE Annual International Symposium on Smart Structures and Materials, Mar, 2001. 